\title{
LA GESTIÓN DE LAS EMPRESAS ANCLAS Y SU INFLUENCIA EN EL DESARROLLO DE NEGOCIOS INCLUSIVOS DEL MAIZ DURO AMARILLO DE LA PROVINCIA DE MANABI - ECUADOR
}

\author{
THE MANAGEMENT OF ANCHORED COMPANIES AND THEIR INFLUENCE ON THE \\ DEVELOPMENT OF INCLUSIVE BUSINESSES OF THE HARD YELLOW CORN \\ OF THE PROVINCE OF MANABI - ECUADOR
}

\author{
Allan Ramiro Sisalema Naranjo \\ Universidad Laica Eloy Alfaro de Manabis \\ Bahía de Caráquez, Ecuador \\ Correo electrónico: \\ allansisalema@hotmail.com
}

[Recibido: 28/09/2018 Aceptado: 17/12/2018]

\section{RESUMEN}

Objetivo: Determinar los fundamentos metodológicos de la Gestión de Empresas Anclas y el Desarrollo de los Negocios Inclusivos. Métodos: Es una investigación de tipo transversal, cuantitativa, explicativa de revisión bibliográfica y documental, muestreo y entrevistas, encuestas, análisis de fiabilidad, pruebas de hipótesis, coeficiente de correlación Spearman. Para el procesamiento y análisis de la información se utilizó el software SPSS.20. Resultados: Según nuestra investigación, la hipótesis general era la existencia de influencia positiva entre la gestión de las empresas anclas y el desarrollo de negocios inclusivos del maíz duro amarillo de la provincia de Manabí- Ecuador, ya que se generó un valor $\mathrm{RHO}=0,893^{*}$ con un valor $\mathrm{p}=0,000<0,05$ responde que la hipótesis es verdadera, por cuanto se ha verificado la relación significativa entre la variable $(X)$ Gestión de las Empresas Anclas e (Y) Desarrollo de Negocios Inclusivos utilizando el método correlación de Spearman, se obtuvo como resultado que existe un alto grado de relación o significativa asociación positiva. Conclusiones: Podemos establecer que la investigación nos concedió unir las secciones más pobres de la sociedad a la cadena de valor de la empresa, permitiendo que este quintil de la sociedad se transforme en un stakeholders, que permite un vínculo de transferencia de tecnología de la producción y guías de herramientas administrativas.

Palabras clave: Empresas anclas, negocios inclusivos, stakeholders, alianzas estratégicas, sostenibilidad.

\section{ABSTRACT}

Objective: To determine the methodological foundations of the Management of Anchor Companies and the Development of Inclusive Businesses. Methods: it is a cross-sectional, explanatory quantitative investigation of bibliographic and documentary review, sampling and interviews, surveys, reliability analysis, hypothesis testing and Spearman correlation coefficient. For the processing and analysis of the information, the SPSS.20 software was used. Results: According to our research, the general hypothesis was the existence of a positive influence between the management of anchor companies and the development of inclusive businesses of yellow hard corn in the province of Manabí-Ecuador. Since an $\mathrm{RHO}$ value $=0,893$ was generated ${ }^{*}$ with a value $p=0,000<0,05$ reveals that the hypothesis is true, since the significant relationship between the variable $(X) \mathrm{Ma}$ nagement of the Anchor Companies and $(Y)$ Development of Inclusive Businesses using the Spearman correlation method was verified. It was obtained as a result that there is a high degree of relationship or significant positive association. Conclusions: we can establish that the research allowed us to link the poorer sections of society to the value chain of the company. This allowed this quintile of the society to become a stakeholder, which allows a link of technology transfer of production and administrative tool guides.

Keywords: Anchor companies, inclusive businesses, stakeholders, strategic alliances and sustainability. 


\section{INTRODUCCIÓN}

La inclusión social aparece como un concepto propio de la responsabilidad social corporativa, paso importante en el mecanismo global de la sostenibilidad y el cumplimiento de las metas de milenio. La lucha contra la pobreza extrema se acompaña de la derrota del analfabetismo, el aseguramiento de la equidad y la salud, la sostenibilidad ambiental y la cooperación internacional para su obtención. En la lucha global contra la pobreza el sector empresarial se constituye en un factor esencial, en la medida en que puede ampliar la base económica de la sociedad mediante la creación de empleo y la generación de riqueza.

El punto de partida es la noción de la Base de la Pirámide (BdP), que se refiere "al segmento de la población con ingresos anuales inferiores a 3260 dólares americanos (USD), compuesto por más de 4000 millones de personas en el mundo" Montaño y Montesinos (2011, p. 89). El valor transaccional de esta cantidad de familias pobres ha atraído la atención de muchas empresas, que observan en ello una oportunidad de hacer negocios, incorporando esta porción de la población, (que se ubica en la base de la pirámide), en su cadena de valor. En otras palabras, se trata de implicarlos en la dinámica económica de la economía global. Estas iniciativas no solamente contribuyen a la reducción de la pobreza, sino también generan nuevas vías para el acceso a oportunidades para la generación de ingresos y del autoempleo. La innovación en ideas colabora eficazmente en la competitividad de las redes de valor, bajo el acompañamiento de las medianas y grandes empresas.

La gestión de una negociación inclusiva nace de la planificación, ejecución y seguimiento de alianzas sociales, con aquellos que se encuentran en los niveles de bajos ingresos. No es fácil poder concertar este tipo de coaliciones, ya que los desafíos que impone su sostenibilidad en el tiempo pueden imponer fragilidad. Sin embargo, queda claro que se trata de una negociación gana-gana, donde se comparten conocimientos, recursos y experiencias, ofreciendo entusiasmo en una primera instancia, la cual debe aprovecharse al máximo, pero siempre actuando con transparencia y eficiencia.

El enfoque de Alianza para Negocios Inclusivos (s.f.) Se basa principalmente en cinco pilares: Negocios Inclusivos, Políticas Públicas Inclusivas, Inclusión Financiera, Empoderamiento Social, Cambio Climático.
Aunque cada enfoque puede gestionarse por sí mismo, la combinación de éstos crea un enfoque integral para el desarrollo económico y social, vinculando a los sectores privados, públicos y civiles para alcanzar objetivos comunes.

Antes de entrar al detalle de la definición de los negocios inclusivos, se debe dar un nuevo abordaje a la conceptualización de la pobreza, ya que la forma como definimos un problema, condiciona la estrategia de resolverlo. James Austin anuncia que la "necesidad de nuevos marcos conceptuales con respecto a la pobreza es hoy tan urgente como nunca" Austin y Chu (2006, p. 3) lo cual sugiere abordar las necesidades desde el estudio de su etiología, esperando un mejor análisis y posterior reflexión de las alternativas de solución.

"Tradicionalmente, la pobreza se define como la carencia de recursos materiales" Gutiérrez y Lobo (2006, p. 41). Esta visión de la pobreza dio alcance a políticas de carácter asistencial, ya que se trataba simplemente de dar lo que se carecía y relegando la posibilidad de generar estos recursos por sí mismos. Esta práctica ha llevado a establecer un fuerte estado de conformidad de lo que se tiene, y por consiguiente la ausencia de un espíritu luchador y recursivo que es preciso para el autoabastecimiento de recursos.

A nivel de la actividad productiva agrícola y pecuaria el Estado ecuatoriano ha invertido en semillas, capacitación, dotación de tierras, maquinaria, prestamos, asesoramientos y controles en precios y en algunos casos el cierre de importaciones de productos como el maíz beneficiando a nuestros agricultores y que actualmente lo cual ha llevado al incremento la producción de maíz en el país y el cual se moviliza internamente favoreciendo a toda la cadena de la producción, comercialización y agro industrialización de esta gramínea.

"En el Ecuador las mayores provincias en la producción de maíz duro amarillo, son Guayas y Los Ríos con 19 \% y 40 \% de participación nacional aproximadamente" Peña, Andrade, y Landy (2018, p. 3) cabe indicar que las otras provincias de la Costa también producen maíz pero en cantidad que no llegan promedios de 10 ha por agricultor, pero se da a entender que es una producción constante año tras año y con los nuevos cambios y aportes al desarrollo de este cultivo está en progresivo incremento.

Debido a los cambios que se distinguen en la zona desde hace 6 años se plantea el análisis de la cadena de 
la comercialización del maíz duro en la provincia de Manabí, para determinar las perspectivas de cada uno de los componentes de la cadena y de demostrar que el sector maicero de esta región puede llegar a un modelo de optimización de la comercialización de otras gramíneas que se dan en la zona y poder ser competitivos en mercados nacionales e internacionales.

Red Productiva (USAID), como parte de su misión de fortalecimiento de las Agencias de Desarrollo Económico Regional (ADETS) para mejorar su capacidad técnica y niveles de sostenibilidad, apoyará a las ADETs a implementar programas de fomento productivo en las provincias que son parte, en los sectores de agro-negocios y turismo.

El año 2010 el Consejo Provincial de Manabí diagnosticó las siguientes necesidades del subsector:

> Fortalecer los procesos de transferencia tecnológica hacia los productores para que mejoren su productividad.

> Promover mecanismos alternativos de financiamiento, que permitan que los productores acceder a mejores condiciones de crédito productivo, de acuerdo a las necesidades de liquidez para implementar un eficiente paquete tecnológico en sus cultivos. Necesidad de superar la falta de riego como factor limitante para la producción.

> Generar interacción directa de productores y mercado, reduciendo la cadena de intermediación, promoviendo un mercado estable con condiciones formales de comercialización con la intervención de una empresa ancla.

> Fortalecer asociatividad como una estrategia que permita consolidar volúmenes y bajar costos de transacción.

Es claro para el Consejo Provincial de Manabí identifica la necesidad urgente de diseñar una estrategia de intervención que permita articular la oferta con la demanda. La propuesta genera soluciones a varios factores críticos definidos en el proceso productivo, como son: el acceso al financiamiento ágil y seguro, además de la implementación de un paquete tecnológico probado, que asegure niveles de productividad rentables a los agricultores, así como la comercialización de la producción bajo la modalidad de integración a la empresa ancla. La solución de estos factores promoverá el fortalecimiento de la cadena productiva, la generación de empleo, incremento de in- gresos de los agricultores y el aumento de inversión en la cadena de maíz en la Provincia de Manabí, conforme el objetivo planteado.

PRONACA S.A. es la empresa más grande del Ecuador, que interviene en los sectores agropecuarios y de balanceados, en el que consume el $60 \%$ de la producción anual de maíz en el país, además de otras materias primas para la elaboración de alimentos animales. La empresa maneja un sistema de integración de productores y contratos de producción y comercialización, cuya principal fortaleza es la implementación de paquetes tecnológicos probados y la dotación de asistencia técnica constante durante los procesos productivos de los integrados. El Consejo Provincial está consciente que los pequeños productores de la provincia no cuentan con los suficientes recursos e instrumentos necesarios para ingresar de manera inmediata al plan de integración de la Empresa Ancla, razón por la cual plantea que de manera conjunta con PRONACA se realice una transferencia de paquete tecnológico y modelo de negocio que permita la integración estable de los productores al Plan Maíz de la Empresa.

Se identifican obstáculos que en esta investigación deberán ser analizados y en su desarrollo brindar soluciones para su implementación y alcanzar metas propuestas.

> Limitados programas de transferencia tecnológica, que sirva para mejorar las prácticas de los productores de cara a articularse a la cadena de valor de la empresa ancla.

> Escaso conocimiento sobre el manejo de un fondo rotativo, que sirva como instrumento que facilite el acceso al paquete tecnológico a los beneficiarios tendientes a mejorar su productividad por hectárea.

Según Maldonado (2014) "el término empresa ancla es nombrado por programas de gobierno de países", sin embargo, se asemeja que preexiste muy escasos escritores que nombran la definición en sí, en efecto a continuación se enfatiza en aquellas hipótesis concernidas, con el objetivo de identificar su relación y hallar aquellos elementos relevantes que consienten a las instituciones conseguir la finalidad de ser empresas anclas.

En este sentido, el Manual Negocios Productivos hace énfasis que la Organización de las Naciones Unidas (ONU) por medio del Esquema de Impulso de Proveedores visualiza ésta terminología como algo muy significativo USAID (2009). 
La empresa ancla lucra de la aplicada cadena fructífera para mejorar su eficiencia como un puntal al perfeccionamiento de los negocios pequeños y medianos que informan en la transformación del producto concluyente. Esto podría establecer vínculos de periodos largos en saber de perfeccionar sus contenidos en un fin común. El estudio de la cadena de producción del negocio permite mitigar los costes de la cadena productiva, valorando de forma vertical la cadena de producción, puede descubrir circunstancias que fácilmente no se observan. En el específico aspecto de los proveedores con la empresa madre (ancla) se deberá establecer las políticas comerciales transparentes que evidencie beneficios mutuos, esto permitirá una mayor confianza para ambos negociantes.

Cordero (2009) referente al tema Erradicando Pobreza mediante Beneficios manifiesta lo siguiente:

La ocasión de negocios en la base de la pirámide, esboza un modelo de negocios fructuoso, que sirve a las sociedades más necesitadas, en donde se plantea varias interrogantes, como las subsiguientes: ¿Qué pasa si movemos los recursos, la progresión y los pesos de las grandes firmas para establecer colectivamente soluciones a los problemas de la base de la pirámide?, ¿Por qué no usar el musculo financiero de las grandes firmas, su conocimiento y el compromiso del Estado con las comunidades que necesitan ayuda?, ¿Por qué no fundar remedies originales de manera unida? (p. 8-11).

Frente a estas interrogantes bosqueja sus máximas, encaminando los empujes de las grandes empresas en la base de la pirámide, es decir en la creación unida de un mercado en torno a las carencias de los pobres, quienes le incumben convertirse en consumidores activos, lo cual puede manifestarse en el alivio de la pobreza. Con esta lógica, declara que, el problema de la pobreza es una dificultad que se puede solucionar, se precisan orientaciones nuevas y creativas para transformar la pobreza en oportunidad para todos los actores.

En este aspecto Schmidheiny (s/f) señala que este modelo tiene otra variante, no desde el punto de vista del consumidor, como lo anotado, sino desde el lado de los proveedores, y es la de incorporar a la economía mundial al gran grupo de proveedores, también compuesto por millones de talentosos creadores de bienes y servicios, a pequeña escala; que, con el concurso de empresas madres o anclas, pueden aprovecharse de mayores mercados, y así mejorar su posición económica, constituyéndose también en un alivio servicios básicos, se puede estimar que cualesquiera de los favores trascienden claramente perceptibles. Según el autor, cabe resaltar el papel de las pequeñas y medianas empresas PYMES, como promotores de la invención y el trabajo.

Por lo antes mencionados esta investigación se declara que la pregunta de investigación es, ¿En qué medida la gestión de las empresas anclas se relaciona con el desarrollo de negocios inclusivos del maíz duro amarillo de la provincia de Manabí- Ecuador? Tenemos como posible hipótesis que la gestión de las empresas anclas se relaciona con el desarrollo de negocios inclusivos del maíz duro amarillo de la provincia de Manabí- Ecuador.

La investigación tiene el objetivo de probar en qué medida la gestión de las empresas anclas se relaciona con el desarrollo de negocios inclusivos del maíz duro amarillo de la provincia de Manabí- Ecuador.

\section{MATERIAL Y MÉTODOS}

Hernández, Fernández y Baptista (2010) refieren que el tipo de la investigación está determinado por su nivel y método. En cuanto al nivel, de acuerdo a la naturaleza del problema planteado y objetivos propuestos, la investigación es de tipo sustantiva explicativa y aplicada con respecto al método empleado, es decir de acuerdo a la estrategia que se utilizó para comprobar las hipótesis formuladas, responde al tipo cuantitativa, transversal y retrospectiva asimismo esta investigación clásica cuantitativa corresponde al tipo analítico, descriptivo, correlacional y no experimental. La presente investigación muestra un diseño correlacional ya que se muestra como fin conocer el vínculo o nivel de asociación que se presenta entre dos o más definiciones, clases o variables en una circunstancia en específico. En cierto grado tiene un valor explicativo parcial debido a que el hecho de conocer que dos definiciones o variables se vinculan, brinda datos explicativos (Hernández-Sampieri, Fernández-Collado, \& Baptista-Lucio, 2014).

El tratamiento de la investigación comprendió la elaboración del marco teórico (marco filosófico, antecedentes nacionales e internacionales y bases teóricas) necesario para el entendimiento de la indagación realizada. Se realizaron entrevistas y se practicaron dos cuestionarios al usuario interno y externo. 
En Ecuador existen 47 empresas que trabajan bajo este esquema de negocios con el respaldo del Servicio Holandés de Cooperación al Desarrollo (SNV), que llegó con la propuesta hace dos años. De estas, 20 están en pleno funcionamiento. Es el caso de Pronaca, Agripac, Supermercados La Favorita, General Motors, Toni, etc. Se calculó la validez de consistencia interna del instrumento para la variable X e Y, mediante el coeficiente Alfa de Cronbach el cual denota una elevada consistencia interna entre los ítems que conforman el cuestionario, ya que el resultado del cálculo correspondiente para X fue de 0,812 y para $\mathrm{Y}$ fue de 0,880 lo que evidencia que las preguntas del cuestionario contribuyen de manera significativa a la definición de los conceptos que se desean investigar, ya que cuando el coeficiente se aproxima a uno, el instrumento es muy confiable para la presente investigación.
Se establecieron dos segmentos importantes en las encuestas, el primer grupo se refiere a los directivos de las 20 Empresas respaldadas por lo tanto no se calculará el tamaño de la muestra, por cuanto el número de entrevistas es mínimo. El segundo grupo son los tres principales stakeholders como socios, clientes y empleados, se establece un muestreo no aleatorio y la cantidad establecida para el presente estudio es de 156 encuestas.

\section{RESULTADOS}

A continuidad se explican los resultados de la encuesta efectuadas a los miembros de los diferentes sectores del conocimiento.

¿Indique cuánto ha mejorado la comercialización de su producto el Modelo de Negocio Inclusivo?

Tabla 1.

Distribución de la muestra según el nivel de mejoramiento de comercialización del producto según modelo de negocio inclusivo.

\begin{tabular}{rcc}
\hline Categorías & Frecuencia & Porcentaje \\
ALTO & 40 & $26,66 \%$ \\
MEDIO & 80 & $53,33 \%$ \\
BAJO & 30 & $20,00 \%$ \\
\hline TOTAL & 150 & $100 \%$
\end{tabular}

Fuente: Elaboración propia.

Como se puede evidenciar en la tabla 1 , los negocios que están vinculados a empresas anclas han mejorado su comercialización en términos de alto impacto con un $26,66 \%$ y medio $53,33 \%$ debido a la política inclusiva del Gobierno Ecuatoriano que ofrece prioridad a estos mercados permitiendo la transferencia de conocimiento y tecnología, los de bajo impacto se deben a las áreas distantes donde aún los medios de tecnologías son limitados y poco accesibles.

¿Este tipo de prácticas inclusivas le han permitido posicionarse en nuevos mercados? 
Tabla 2.

Distribución de la muestra según el posicionamiento en nuevos mercados.

\begin{tabular}{ccc}
\hline Categorías & Frecuencia & Porcentaje \\
PENETRANTE & 56 & $26,66 \%$ \\
MODERADO & 75 & $53,33 \%$ \\
BAJO & 19 & $20,00 \%$ \\
\hline TOTAL & 150 & $100 \%$
\end{tabular}

Fuente: Elaboración propia.

La tabla 2 manifiesta que, continuando una visión de avance económico moderado por medio de una estrategia de Mercados Inclusivos construida y desarrollada conjuntamente, nuestros países pueden ser actores en el adelanto de mercados verdes e inclusivos, comprometidos en la mejora de la situación del continente y generar un modelo que permita su aplicación en otras áreas del planeta.

¿Se establecen nuevas alianzas que ayuden a materializar el objetivo de mejorar la calidad y cantidad requeridas por la empresa compradora?

Tabla 3.

Distribución de la muestra según las alianzas estratégicas.

\begin{tabular}{ccc}
\hline Categorías & Frecuencia & Porcentaje \\
ELEVADO & 55 & $36,66 \%$ \\
INTERMEDIO & 78 & $52,00 \%$ \\
ESCAZO & 17 & $11,33 \%$ \\
\hline TOTAL & 150 & $100 \%$ \\
\hline
\end{tabular}

Fuente: Elaboración propia. 
El argumento de las coaliciones fructuosas es muy importante porque escasos sectores del país, sean agrícolas, manufactureros o de servicios, mediante los negocios inclusivos les han ofrecido la ocasión a los más pequeños de ser dueños de un proceso productivo; de manera, que aquí no solamente se les ha adiestrado en sembrar, sino que, se les ha ayudado a comprar e identificar el paquete tecnológico, a la articulación a la empresa que demanda el producto y a ser más eficientes y eficaces en el manejo de los recursos .

Evidenciamos según la tabla 3 como va incrementado el nivel de alianzas, $36 \%$ de los encuestados confían plenamente en este proceso y un $52 \%$ están en proceso de firmar dándole la total apertura a las alianzas estratégicas, el $11,33 \%$ determina que tienen limitada información sobre el tema debido a su ubicación geográfica y condiciones de acceso a la tecnología.

Tabla 4.

Distribución de la muestra según las alianzas estratégicas: Rho Sperman

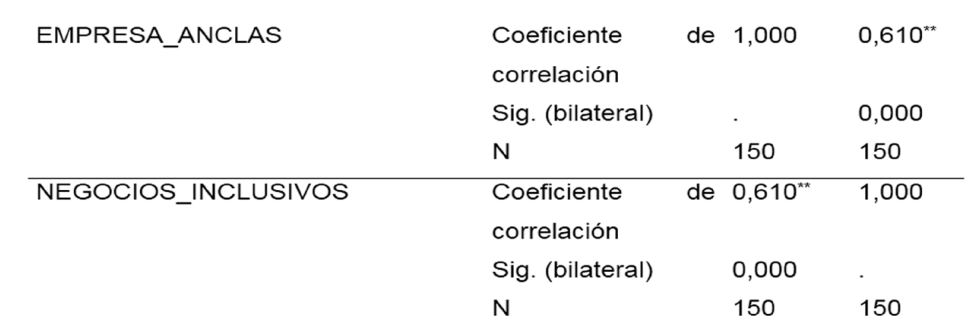

. La correlación es significativa al nivel 0,01 (bilateral).

Fuente: Elaboración propia.

Como resultado, se puede establecer que la hipótesis es verídica, por cuanto se ha probado con alto grado de significancia que la variable $(\mathrm{X})$ influye sobre la variable $(\mathrm{Y})$, razón primordial de la demostración de la hipótesis.

\section{DISCUSIÓN}

Según nuestra investigación, la hipótesis general era la existencia de influencia positiva entre la gestión de las empresas anclas y el desarrollo de negocios inclusivos del maíz duro amarillo de la provincia de Manabí- Ecuador, ya que se generó un valor $\mathrm{RHO}=0,893^{*}$ con un valor $\mathrm{p}=0,000<0,05$ que responde que la hipótesis es verdadera, por cuanto se ha verificado la relación significativa entre la variable (X) Gestión de las Empresas Anclas y (Y) Desarrollo de Negocios Inclusivos utilizando el método correlación de Spearman, se obtuvo como resultado que existe un alto grado de relación o significativa asociación positiva. 
Conforme a Cordero (2009) en su tesis titulada como “emprendimientos inclusivos" se presentó un breve bosquejo sobre los Emprendimientos Inclusivos, tomando como punto de partida un análisis conceptual, tema que lo trataremos en esta investigación, se llegó a recopilar una serie de buenas prácticas de empresas nacionales e internacionales que están desarrollando este modelo, que los hemos llamado casos de emprendimientos inclusivos, no se ha podido profundizar en el estudio, sino más bien se ha tratado de recopilar una interesante base de casos, algunos de ellos con resultados ya visibles, en otros, con resultados a ser visibles el mediano plazo.

Coincidimos con los estudios anteriormente nombrados, que el Desarrollo de Negocios Inclusivos es una herramienta fundamental para el ámbito administrativo de toda corporación y, en efecto, las empresas anclas generan un buen encadenamiento productivo que posibilita a los proveedores la contribución exitosa con mejor unificación de sus cadenas productivas.

Se recomienda al Estado como a las organizaciones no gubernamentales, empresas y sociedad civil trabajen en conjunto para promover la implementación de la gestión de empresas anclas y desarrollo de negocios inclusivos del maíz duro amarillo, resolviendo sus limitantes. En el Estado Ecuatoriano, es crucial que este genere políticas públicas que promuevan e incentiven la asociatividad e inclusión en los negocios de las empresas en más de una de sus cadenas, para lograr un mayor impacto. Es importante desarrollar programas de motivación para empresarios que temen progresar con sus microempresas y de manera paralela se desarrollen de autoestima, así mismo también desarrollar capacitaciones de trabajo en familia, debido a que la mayoría de las empresas trabajan con su grupo familiar y ellos influyen mucho en la actitud del empresario.

\section{REFERENCIAS BIBLIOGRÁFICAS}

Austin, J., \& Chu, M. (2006). La empresa privada y los sectores de bajosingresos. Revista Harvard Review of Latin America, 6(1), 3-5.

Banco Central del Ecuador. (2015). Estadisticas del sector Agropecuario. https://www.bce.fin.ec/index.php/ estadisticas-economicas2o
Cordero, J. (2009). Emprendimientos Inclusivos. Cuenca: Universidad de Cuenca.

Gutiérrez, R., \& Lobo, I. (2006). Es posible aliviar la pobreza a través delos negocios? Revista.Harvard Review of Latin America, 6(1), 39-41.

Licandro, O. (2013). Modelos para el análisis de los negocios inclusivos: construcción mediante el estudio de casos. Revista de Estudios Interdisciplinarios en Ciencias Sociales, 15(1), 32-48.

Maldonado, G. (2014). Empresa ancla y el desarrollo de las MIPYMES (proveedoras) Facultad Latinoamericana de Ciencias Sociales Sede Ecuador Departamento de Desarrollo Ambiente y Territorio. Ecuador. http://hdl.handle. net/10469/7471

Prahalad, C. (2005). La oportunidad de negocios en la base de la pirámide. Un modelo de negocios rentable, que sirve a las comunidades más pobres. Wharton School Publishing. 12, 67-91.

Peña, N., Andrade, E., \& Landy, L. (2018). Análisis del sistema de comercialización de la producción de maíz para mejorar los ingresos de los pequeños productores del recinto aguas frías de Medellín, Cantón Ventanas, provincia de Los Ríos. Ecuador: Revista Observatorio de la Economía Latinoamericana.

Porter, M., \& Kramer, M. (2006). Estrategia y sociedad. Harvard Business Review, 84(12), 42-56. Obtenido de http://www.fundacionseres.org/Lists/Informes/Attachments/12/Estrategia\%20y\%20Sociedad.pdf

Montaño, N., \& Montesinos, Y. (2011). El impacto sobre las empresas en lo referente a la responsabilidad social empresarial y negocios inclusivos, periodo 2010-2011. Cuenca: Universidad de Cuenca.

Servicio Holandés de Cooperación al Desarrollo. (2008). Un enfoque empresarial sobre negocios con el mercado de la mayoría: Proyecto mapeo del sector privado. $\mathrm{Ob}$ tenido de https://goo.gl/wQbE3q

Sostenible, C.E. (2008). Alianza para los Negocios Inclusivos. Los negocios inclusivos en Colombia. Colombia. 